\title{
INTRINSIC SELF SENSING CONCRETE
}

\author{
Vimarsha B.R ${ }^{1}$, T Soumya ${ }^{2}$, Rohit Gainole ${ }^{3}$ \\ ${ }^{I}$ Graduate, Structural Engineering, MVJCE, Karnataka, India \\ ${ }^{2}$ Assistant Professor, Civil Department, BMSCE, Karnataka, India \\ ${ }^{3}$ Graduate, Structural Engineering, MVJCE, Karnataka,, India
}

\begin{abstract}
Structural Health Monitoring (SHM) of concrete structures is now a very important technology in order to ensure that the structure is safe and sound. But there are many disadvantages in SHM. Hence it calls for much better and a very simple approach, therefore it's our responsibilities as Structural Engineers to find solutions to these quagmires. This research concentrates on finding solution to the limitations of using sensors by making use of the advanced Nanotechnology. Carbon Nanotubes are used as a Self-Sensing tool to achieve Intrinsic Self-Sensing ability of the concrete or cement mortar. The indigenous electric resistivity parameter of Carbon Nanotubes is made use of effectively in developing the Intrinsic Self Sensing ability of the concrete or cement mortar. Copper rods are used to achieve resistance property of the CNT's. Two probe method is made use of for the connections to get the resistance. Resistance is tabulated using multimeters. And the results are tabulated. Experimental results show excellent electric resistivity response when Carbon Nanotubes are used in the cement mortar specimens.
\end{abstract}

Keywords:Structural Health Monitoring, Intrinsic Self Sensing Concrete,Carbon Nanotubes, Nanotechnology, Two Probe Method etc...

\section{INTRODUCTION}

During the design of a structure it should be designed in such a way that it must provide maximum resistance to all possible unexpected damages. Therefore a structure must be monitored every time in order to prevent these damages and ensure safety for the people living in those structures. So Structural Health Monitoring is one approach to get rid of these damages. Structural Health Monitoring includes use of embedded sensors like optical fibre sensor, stress or strain gauge sensors, electrical resistance sensors, FBG sensors, smart aggregate sensors, smart materials, piezoresistive ceramic sensors which are placed in some critical points were the damages are likely to occur. But these sensors have their own drawbacks like, monitoring occurs only in and around the critical points, very less durability, expensive and complex analysis of results since high end instruments are used, when these sensors get damaged inside the concrete specimen, they fail and are no longer available for sensing. Therefore it becomes necessary for a simple and better approach and it's our bonded responsibility as Structural Engineers to solve these problems. So the best and ideal solution is to make the concrete itself Self-Sensing or SelfMonitoring without the aid of any sensors or any complex arrangements. Therefore the name "Self Sensing Concrete". Since this process happens inside the specimen it is named as "Intrinsic Self Sensing Concrete".

Self-sensing is the phenomenon in which the concrete itself monitors its health when there might be unexpected potential threats for the concrete structure, during these life threatening situations its tougher for the engineer to provide necessary solutions in very short decision making time. So if the concrete structures is enabled with a Self-Sensing ability, it can intimate the occurrence of the unexpected failures at the earliest. Therefore it becomes very easier for the damage detection prior to the damage has actually occurred which is a very good sense of responsibility a Structural Engineer should have.

This project concentrates on finding solutions to the limitations of sensors, by making use of the advancement in Nanotechnology.

Use of Carbon Nanotubes flexibly for various applications is recommended for this study. Carbon Nanotubes have the best properties like very good mechanical properties, higher young's modulus, and excellent electrical and electromechanical properties. Therefore it helps us to explore the vast variety of properties of CNT's and make the best use of them.
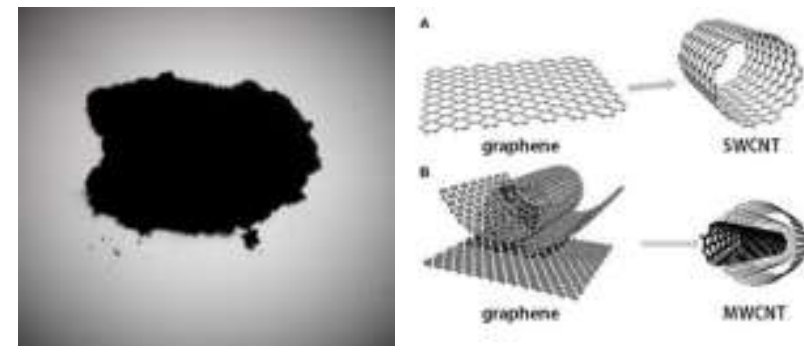

Fig 1:Image of Multiwalled Carbon Nanotubes 


\subsection{Objectives}

- To achieve the sensing ability of the cement mortar specimen.

- To ensure the electric conductive property of the cement mortar specimen with the action of CNT's.

- To detect the micro and Nano level cracks.

- To detect the failure before it actual occurs.

\section{REVIEW OF LITERATURES}

Dr.Xun Yu and Dr.Eil Kwon (2012), In this research project carboxyl group multi walled carbon nanotubes are used. To conduct electric current between external sources to cement composite, Stainless steel meshes of $6 \mathrm{~mm}$ diameter with opening of $1.25 \times 1.25 \mathrm{~cm}$ were used as electrodes. Both meshes are placed inside the composite with $1 \mathrm{~cm}$ apart. Cement mortar of 5 cubic feet is prepared. The piezoresistive response is checked for both lab test and road test for traffic flow detection. Electrodes are kept perpendicular to compression load while testing for resistivity. Resistance is recorded with respect to time factor while applying compression and impact loading. This paper also explains the comparative results of piezoresistance for different concentration of MWCNTs by having higher response for $0.1 \%$ compare to $0.05 \%$ and $1 \%$. Hardiksolanki, (2017).The paper focus on enhancement of conducting property of cement mortar by adding MWCNTs.They have used $0.75 \%$ of CNT by weight of cement and dispersed in distilled water using same amount of lignosulphonates as surfactant because of its easy availability and cheap. They used Sonication method for dispersing and casted into $70 * 70 * 70 \mathrm{~mm}$ mortar cube. Then its tested for conductive property by passing 5 volt DC current and also they found out its 7th day compressive strength which is approximately 28th day compressive strength of conventional concrete preparing samples of different proportions of MWCNTs added and the tests conducted for cyclic and dynamic tests. Nano-modified cement-based sensors represent an innovation in civil engineering. D - Alessandro, (2017) This paper has presented an experimental research work on the use of cement based sensors doped with multi-walled carbon nano-tubes as smart embedded reinforced sensors for static and dynamic monitoring of reinforced concrete components. The experimental campaign started from the electrical and electrochemical characterization of a meaningful number of samples in order to investigate the repeatability and accuracy of sensors' behaviour in unloaded conditions, as well as under the application of both static and dynamic mechanical loads.

\section{MATERIALS AND METHODOLOGY}

\subsection{Background}

Structural Health Monitoring includes use of embedded sensors like optical fibre sensor, stress or strain gauge sensors, electrical resistance sensors, FBG sensors, smart aggregate sensors, smart materials,piezoresistive ceramic sensors which are placed in some critical points were the damages are likely to occur. But these sensors have their own drawbacks like, monitoring occurs only in and around the critical points, very less durability, expensive and complex analysis of results since high end instruments are used, when these sensors get damaged inside the concrete specimen, they fail and are no longer available for sensing. Therefore it becomes necessary for a simple and better approach and it's our bonded responsibility as Structural Engineers to solve these problems. So the best and ideal solution is to make the concrete itself Self-Sensing or SelfMonitoring without the aid of any sensors or any complex arrangements. The methodology adopted in this research is as explained, surfactant wrapping of CNT's using surfactant whereSodium Lauryl Sulphate is used as a surfactant. Since it can't be used directly in the dispersion of CNT's first it must be uniformly dispersed in water. A process is done for dispersion using magnetic stirrer.

Carbon nanotubes is added to the dispersed sodium lauryl sulphate after stirring the solution in magnetic stirrer. Next process is uniform dispersion of surfactant coated carbon nanotubes. For this process en equipment called ultrasonic sonicator is used. Next preparation of cement mortar moulds of size $70 \mathrm{mmX} 70 \mathrm{mmX} 70 \mathrm{~mm}$. And curing it for 24 hours in $\mathrm{s}$ fresh clean salt-free water.

Two Probe Method is adopted in this research. In this process the sample is simultaneously contacted with two electrodes and the conductivity is measured. And the copper rods mesh placed during the casting process is as shown in the following figure.

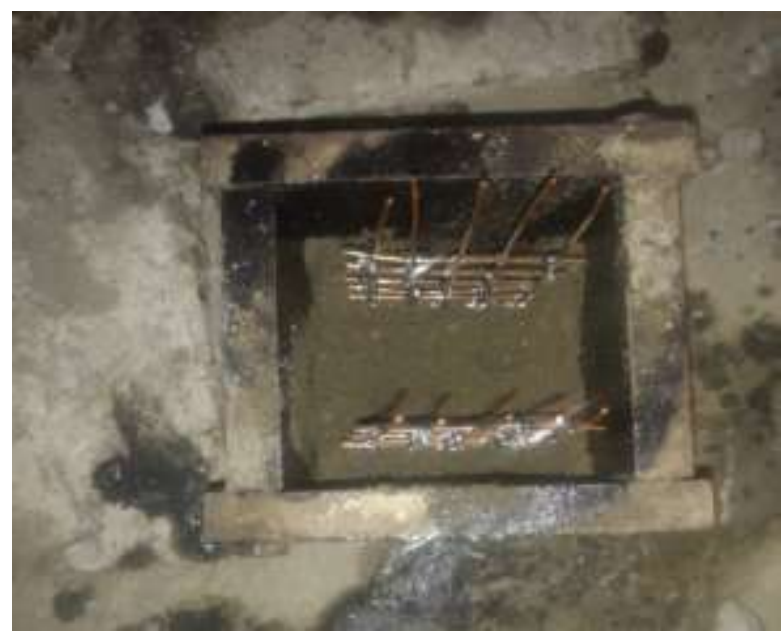

Fig 2: Copper Rods Mesh placed during casting

\subsection{Experimental Campaign}

Electromechanical test means checking out the electrical property of a specimen under mechanical loading simultaneously. In this research the specimen as shown in the figure 1 is subjected to gradual loading kept in the Universal testing machine for compressive strength test along with the electrical test. The experimental setup is as follows: 


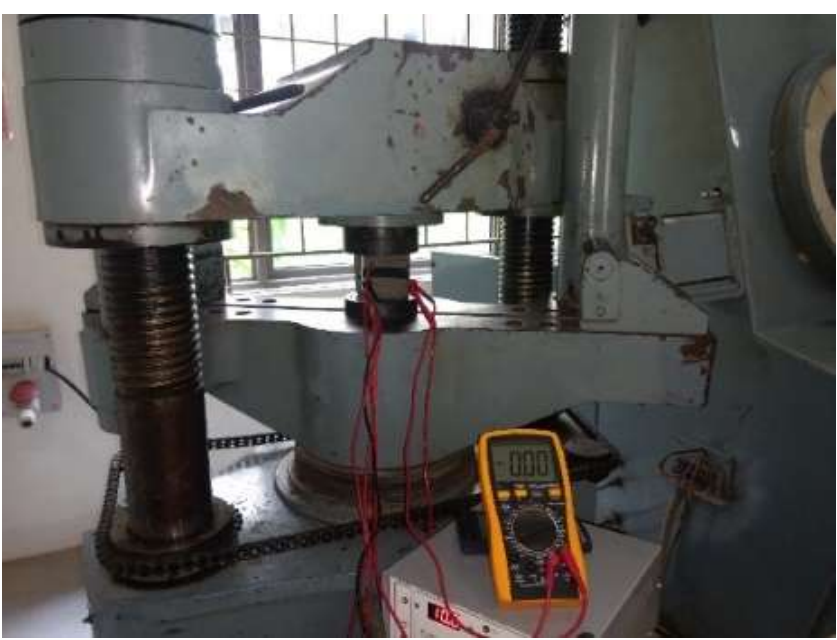

Fig 3: Test setup

- First the specimen is provided with the required wire connections.

- Then those wires are connected to the digital multimeters and a DC supply source.

- Now the entire setup is placed in the UTM for loading.

- Two probe method is adopted for connections. When load is applied the dc source is turned on and readings are tabulated from the multimeters.

- $\quad$ Readings are tabulated until the specimen fails.

\section{TABULATIONS}

Table 1: Tabulations

\begin{tabular}{|l|l|l|l|}
\multicolumn{5}{|c}{ Table 1: Tabulations } \\
\begin{tabular}{|l|l|l|} 
Load \\
$(\mathrm{Kgs})$
\end{tabular} & $\begin{array}{l}\text { Voltage } \\
(\mathrm{V})\end{array}$ & $\begin{array}{l}\text { Current } \\
\left(10^{-3}\right) \text { Amps }\end{array}$ & $\begin{array}{l}\text { Resistance } \\
\left(10^{3}\right) \text { Ohms }\end{array}$ \\
\hline 0 & 16 & 3.60 & 4.44 \\
\hline 2000 & 16 & 3.2 & 5 \\
\hline 4000 & 16 & 3.08 & 5.2 \\
\hline 6000 & 16 & 2.91 & 5.5 \\
\hline 8000 & 16 & 2.71 & 5.9 \\
\hline 10000 & 16 & 2.5 & 6.4 \\
\hline 12000 & 16 & 2.35 & 6.8 \\
\hline 14000 & 16 & 2.24 & 7.15 \\
\hline 16000 & 16 & 1.78 & 9 \\
\hline 18000 & 16 & 1.74 & 9.22 \\
\hline 20000 & 16 & 1.62 & 9.9 \\
\hline 22000 & 16 & 0.73 & 22 \\
\hline
\end{tabular}

\section{CONCLUSION}

Arriving at the important part of the research which is the crucial one, concluding a research in brief as follows.

- The cement mortar specimen was tested for sensing ability successfully.

- It was observed that the micro and nano cracks were decreased due to the CNT's action.

- It was also observed that the compressive strength of the cement mortar specimen was increased by $15 \%$ greater than the normal conventional mortar.

- Failure was unable to be detected before failure.

\section{REFERENCES}

[1] Dr Xun Yu, Dr Eil Kwon-“CNT based self-sensing concrete for pavement Structural health monitoring".

[2] Ajay Raghavan, Seth S, Derreck Barber, Sunny Wicks-"SHM using CNT enhanced composites", 2009.

[3] A D'Alessandro, F. Ubertini -“Self sensing concrete Nano composites for smart structures", 2016.

[4] Tiera Rollins, Erik.T.Thostenson-“Advanced Structural Health Monitoring Using CNT”. 
[5] P.W.Chen,D.D.L Chung-“Carbon Fibre Reinforced Concrete For Smart Structures Capable Of NonDestructive Flaw Detection", 1993.

[6] B Han, Y Wang, S Dong-"Smart Concrete and Structures", 2005.

[7] M.S Konsta-Gdoutos, C.A Aza-"Self-Sensing Concrete Nanotubes and Carbon Nano fibre For Real Time Damage Assessment In Smart Structures", 2014.

[8] X.Yu, E Kwon-“A CNT Cement Composite with Piezoresistive Property", 2009.

[9] F Azhari, N.Banthia-"Cement Based Sensors with Carbon Fibres for Piezoresistive Sensing”, 2012.

\section{BIOGRAPHIES}

VIMARSHA.B.R holds $M$ Tech Degree in Structural Engineering from VTU, Karnataka, India.

T SOUMYA, AssistantProfessor,Department of Civil Engineering,BMS college of Engineering, Bengaluru, Karnataka

ROHIT GAINOLE,holds $M$ Tech Degree in Structural Engineering from VTU, Karnataka, India. 